\title{
Damped periodic motions used in the study of the drag coefficient
}

\author{
Stelian Alaci ${ }^{1,3, *}$, Constantin Filote ${ }^{2,3}$, Daniel Fodorcan ${ }^{4}$, Erwin-Vasile \\ Alexandru $^{1}$, Dumitru Amarandei ${ }^{1,3}$ and Florina-Carmen Ciornei ${ }^{1,3}$ \\ ${ }^{1}$ Stefan cel Mare University, Department of Mechanics and Technologies, 13 University Street, \\ Suceava, Romania \\ ${ }^{2}$ Stefan cel Mare University, Computers, Electronics and Automation Department, 13 University \\ Street, Suceava, Romania \\ ${ }^{3}$ Integrated Center for Research, Development and Innovation in Advanced Materials, \\ Nanotechnologies, and Distributed Systems for Fabrication and Control (MANSiD), 13 University \\ Street, Suceava, Romania \\ ${ }^{4}$ Aalborg University, Design of Mechanical System, Fredrik Bajers Vej 5, 9220 Aalborg, Denmark
}

\begin{abstract}
The paper proposes a method and the related experimental setup for finding the drag coefficient for a body. The device consists in a pendulum materialized by a hinged rod initially in horizontal position. A spherical body immersed in a liquid is attached to the free end of the rod. The restoring force is created by a helical spring. The inertial characteristics of the pendulum can be changed using an additional mass. The differential equation characteristic to the motion of the pendulum is deduced and subsequently integrated numerically. The coincidence between experimental and theoretical data allow for viscosity evaluation.
\end{abstract}

\section{Introduction}

Real fluids can transmit shear stresses between two surfaces between which relative motion exists, due to the property of the fluid - viscosity. The experimental finding of viscosity is still an actual problem. There are a large number of works dedicated to the subject, starting with classical monographs of Stokes [1-2] and ending with the most recent papers and thesis. The subject was approached both from a fundamental sciences point of view [3] and by researchers from applied sciences [4]. A device used in viscosity measurements must satisfy the following condition: to permit the finding of the magnitude of interaction between two rigid surfaces between which there is a liquid when there is imposed motion between the surfaces or, to allow relative motion between the two surfaces when the interaction between them is imposed. The relative motions imposed between the surfaces can be periodical or non-periodical. The shape of the surfaces and the relative motion are the main criteria for viscometer classification [5]. Landau and Lifshitz, [3] present analytical expressions of the calculus of hydrodynamic friction for different combinations of surfaces and relative motions.

\footnotetext{
*Corresponding author: alaci@fim.usv.ro
} 


\section{Principle of proposed device}

The idea of the proposed test rig started from the Nagarajan paper [6]. He uses a viscometer presented in principle in Figure 1, where one of the surfaces is the boundary surface of a gold sphere 1 immersed in an unbounded fluid. The ball is connected to a helical spring 3 and the dynamic system ball-spring is stimulated into oscillation on vertical direction. An electronic transducer 2 registers the motion of the ball and the damping phenomenon is revealed. The causes of damping the author identifies are the drag force and the correction term of inertial force due to the dislocated liquid by the sphere. From the calculus of logarithmic decrement of damping he determines the viscosity of the liquid. Besides the forces met above, other forces are responsible of motion damping [7] like the force describing the history of the motion of the fluid or the correction term for the drag force due to the effect of finite boundaries of the liquid.

The schematic of the new proposed viscometer is presented in Figure 2. It consists of an aluminium horizontal pipe 1 of square cross-section, which can rotate about the joint $O$. A vertical shaft 2 is firmly attached to the rod, having a sphere 3 fastened at the inferior end that can be immersed into the liquid to be studied, 4 . At the other end of the vertical shaft, a body 5 can be fixed eccentrically with respect to the horizontal rod. A supplementary body can be fixed on the rod at a specific distance, on the opposite side of the joint $O$ with respect to the vertical shaft. The function of this body is to change the moment of inertia $\mathrm{JOz}$ of the pendulum with respect to the axis of oscillation and the position of the centre of mass. The gliding body 5 from the vertical shaft has the task to get the centre of mass of the pendulum on the axis of horizontal rod. Additionally, a series of holes with vertical axis are made in the rod and thus the position of the point where the spring 7 is tied up can be modified. The upper end of the spring is fixed in a bushing that can translate vertically. This ensures the possibility that after the spherical body is immersed into the liquid the pendulum will be brought into horizontal position by modifying the spring pre-stressing force. Therefore the moments of weight, pre-stressing and buoyancy forces are balanced. The motion of the pendulum is investigated via a non-contact method. To this purpose, at the end of the rod a small laser stick 8 is fixed and the spot can be watched on a vertical screen $\mathrm{E}$ having a scale $\mathrm{S}$. After the pendulum is set into oscillation, the motion of the spot on the scale from the screen is video-captured and after that the recording is split into frames for identification of extreme values of spot displacement and the index of the frame.

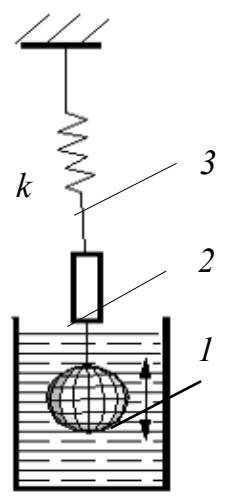

Fig. 1. Viscometer with sphere [6].

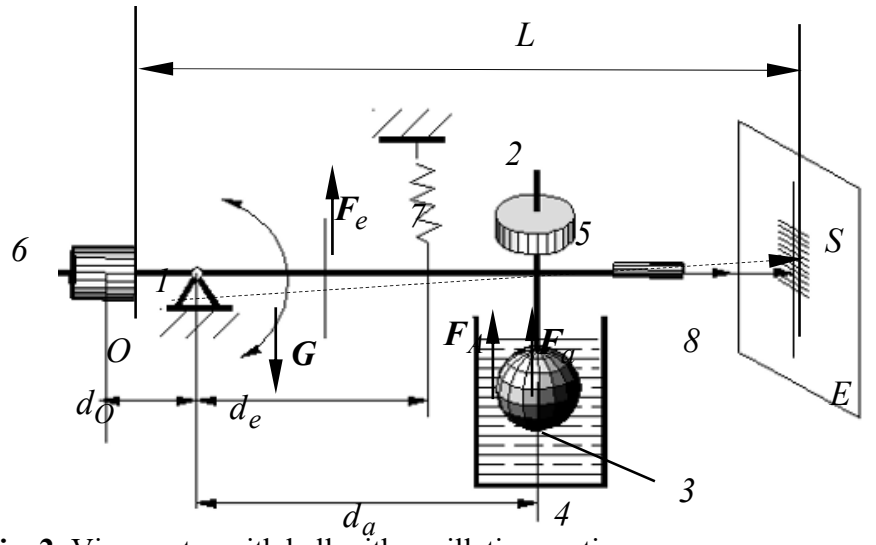

Fig. 2. Viscometer with ball with oscillating motion. 
Compared to the viscometer presented in Figure 1, the proposed device has the advantage that the effects of forces acting upon the pendulum can be controlled, diminished or amplified, by varying the distances of the points of application of forces acting upon the pendulum. The principle of viscosity evaluation is similar to the one used by Nagarajan [6] and refers to the comparison between the damping of theoretical model and the experimental damping. The inertial forces acting upon the ball are [6]:

$$
M=m_{b}+m_{c}+m_{s} / 3
$$

Where $m_{b}$ is the mass of the ball, $m_{c}$ is the mass of the transducer core and $m_{s}$ is the mass of the spring. In our case, $m_{c}=0$ and $m_{s} \cong 0$ since the mass of the spring is insignificant compared to the mass of the ball. The mass of the displaced liquid by the oscillating ball must be considered; Stokes [1] introduced the correction of inertia force:

$$
F_{b}=\left(\frac{1}{2}+\frac{9}{4} \mu\right) m^{\prime} \frac{d^{2} x}{d t^{2}}
$$

where $m^{\prime}$ is the mass of the liquid displaced and $\mu$ is a dimensionless parameter having the expression:

$$
\mu=[\eta T / \pi \rho a]^{1 / 2}
$$

where $\eta$ is the dynamic viscosity, $\rho$ is the fluid density, $T$ is the period of oscillation and $a$ is the radius of the sphere. The total inertial force is given by:

$$
F_{i}=-\left[m_{b}-m^{\prime}\left(\frac{1}{2}-\frac{9}{4} \mu\right)\right] \frac{d x^{2}}{d t^{2}}
$$

Next it is assumed that the period of oscillation of the ball is not affected by the viscous damping. After the ball is completely immersed into liquid, the device is balanced and the pendulum is set to horizontal position using the additional mass 6 . The elastic force acting on the ball is proportional to the spring deformation $x$ and elastic constant of the spring $k_{e}$ :

$$
F_{e}=-k_{e} x
$$

The damping force on the sphere that oscillates vertically in a liquid is given by:

$$
F_{d}=-\frac{9}{2} \frac{\pi \mu M^{\prime}}{T}(1+\mu) \frac{d x}{d t}
$$

and being valid in the domain of velocities that situate the Reynolds number in the range $R e=[40 \div 200]$. The equation of motion of the pendulum is obtained by applying the moment of momentum theorem about the joint centre:

$$
J_{O} d^{2} \theta / d t^{2}=-F_{i} d_{a}-F_{d} d_{a}-F_{e} d_{e}=0
$$

where $J_{O}$ is the moment of inertia of the pendulum with respect to the joint O.

Since the inertial force $F_{i}$ depends on the acceleration, this is taken into account by replacing $J_{O}$ with a new moment of inertia $J_{O}^{\prime}$ that considers the effects of the liquid 
displaced and set into motion by the sphere, as well. With the assumption of small oscillations, $\theta \leq 5^{\circ}$, after some manipulation the equation 7 becomes:

$$
J_{O}^{\prime} \frac{d \theta^{2}}{d t^{2}}+\frac{9}{2} \frac{\pi \mu m^{\prime}}{T} d_{a}^{2}(1+\mu) \frac{d \theta}{d t}+k_{e} d_{e}^{2} \theta=0
$$

The equation 8 is a linear homogenous differential equation with constant coefficients, written under the standard form:

$$
A \ddot{\theta}+2 B \dot{\theta}+C \theta=0
$$

where:

$$
A=J_{O}^{\prime}, d_{a}^{2}, B=\frac{9}{4} \frac{\pi \mu m^{\prime}}{T} d_{a}^{2}(1+\mu), C=k_{e} d_{e}^{2}
$$

With the notations:

$$
\alpha=B / A, \quad \beta=\sqrt{C / A-(B / A)^{2}} \cong \sqrt{C / A}=T
$$

one can attest that between the logarithmic decrement of the model and the experimental damping exponent $\alpha$ the following relation subsists:

$$
\ln \left(\theta_{0} / \theta_{0 n}\right)=\alpha n T
$$

where $T$ is the period and $\theta_{0}, \theta_{n}$ are two angular amplitudes corresponding to the instants 0 and $n T$, respectively. Using relation 11 in equation 12 , the final relation on which the evaluation of viscosity is based is obtained:

$$
\ln \frac{\theta_{0}}{\theta_{0 n}}=\frac{9}{4 T} \pi\left[\frac{\eta T}{\pi \rho a}\right]^{1 / 2} m^{\prime} d_{a}^{2}\left(1+\left[\frac{\eta T}{\pi \rho a}\right]^{1 / 2}\right) \frac{n T}{J_{0}^{\prime}}
$$

\section{Experimental device. Results and discussions}

The experimental set-up employed in laboratory tests is presented in Figure 3 where the liquid used is glycerine. The oscillations of the pendulum are $5^{0}$ visualised on the screen via the laser spot and the geometrical restriction $\theta \leq 5^{\circ}$ can be controlled. The angular oscillations are mapped as linear displacements of the spot on the scale and recorded with a camera in real time. The decrease of the angular amplitude is established on computer, by analyzing the frames of the movie. To eliminate the errors due to the internal friction of the pendulum, the test was also performed in air. The decrease of the amplitude of the pendulum for the two experimental cases was fitted by exponential function of the form $\theta_{0} \exp (-\alpha t)$. The experimental data and the interpolation curves obtained for air and glycerine are given in Figure 4. To eliminate the effect of internal friction, assuming that the values of the damping exponents are small, the following approximation is used: 


$$
\exp (\alpha x) \cong 1+\alpha x
$$

and it results:

$$
\alpha_{g}=\alpha_{g a}-\alpha_{a}
$$

where $\alpha_{g a}$ is the exponent for global experiment, $\alpha_{a}$ is for pendulum oscillating in air and $\alpha_{g}$ is the exponent deduced for the damping produced only by glycerine.

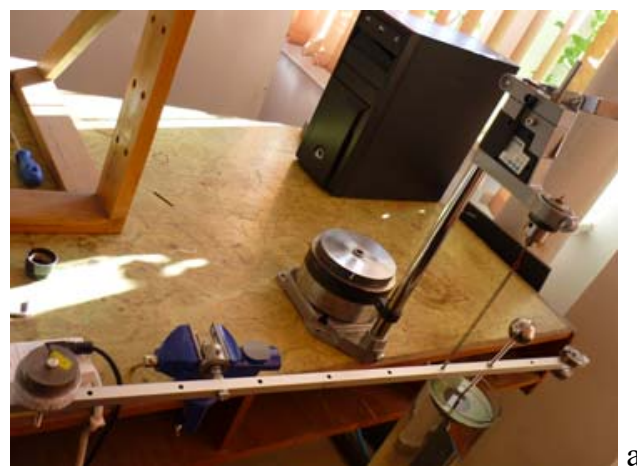

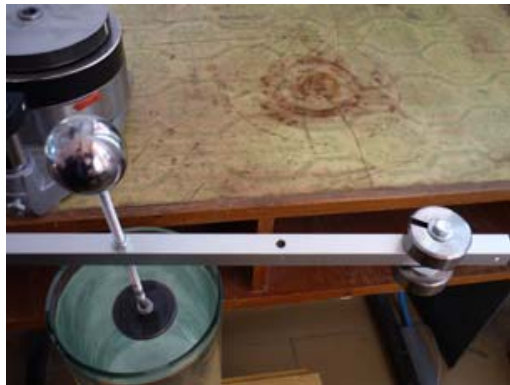

b)

Fig. 3. Experimental set-up: assembly (a) and detail (b).

The theoretical curve due to damping produced only by glycerine is also plotted in Figure 4. For the oscillations in air and liquid, the periods are practically the same $T_{\text {exp }}=1.167 \mathrm{sec}$, as resulting from Figure 5, and the hypothesis that the period is insensitive to the effect of viscosity is confirmed.

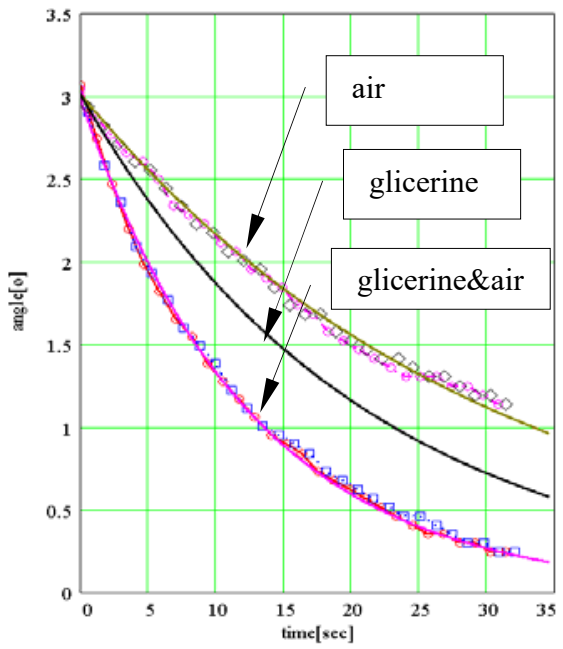

Fig. 4. Amplitude variation-experimental and interpolation curves.

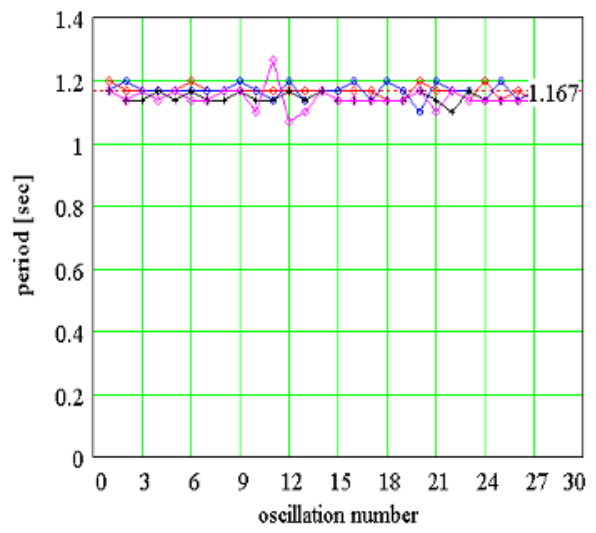

Fig. 5. The period of oscillation is not affected by viscosity.

From the second equation 11 it results that the moment of inertia can be found $J_{O}^{\prime}$ imposing the condition that the period of the model equals the experimental period $T_{\text {exp }}$. 


$$
J^{\prime}{ }_{O}=k_{e} d_{e}^{2} T_{e x p} /\left(4 \pi^{2}\right)
$$

Knowing $J_{O}^{\prime}$ and using the first equation 11, with the condition that the logarithmic decrement of the model is the same as the experimental damping exponent $\alpha$ and taking into account the equation 3 , a transcendental equation that allows finding the viscosity is obtained:

$$
\alpha_{g}-\frac{\frac{9}{4} \pi\left(\eta \frac{T_{\exp }}{\pi \rho^{\prime} a}\right)^{1 / 2} \frac{m^{\prime}}{T_{\exp }} d_{a}^{2}\left[1+\left(\eta \frac{T_{\exp }}{\pi \rho^{\prime} a}\right)^{1 / 2}\right]}{k_{e} d_{e}^{2} T_{\exp } /\left(4 \pi^{2}\right)} .
$$

The experimental parameters were: $a=0.02 \mathrm{~m} ; \rho^{\prime}=930 \mathrm{~kg} / \mathrm{m}^{3} ; J_{0}^{\prime}=0.128 \mathrm{~kg} \cdot \mathrm{m}^{2}$; $k_{e}=70 \mathrm{~N} / \mathrm{m} ; d_{e}=0.25 \mathrm{~m} ; d_{a}=0.45 \mathrm{~m} ; T_{\text {exp }}=1.167 \mathrm{sec} ;$. The damping interpolation exponent $\alpha_{g}=0.048 \mathrm{sec}^{-1}$ results from $\alpha_{g a}=0.081 \mathrm{sec}^{-1}$ and $\alpha_{a}=0.033 \mathrm{sec}^{-1}$. With these values, the value obtained for the experimental dynamic viscosity is $\eta=0.977 \mathrm{~Pa} \cdot \mathrm{s}$, in very good agreement with the values from literature.

\section{Conclusions}

The present paper proposes a method and device for finding the viscosity of a fluid. The method is based on the viscometer with vertical oscillations of a ball and spring system. The effect of damping forces of the fluid upon the oscillations of the ball is characterized by logarithmic decrement of oscillations, accepting thus an exponential function for the decrease of oscillation amplitude. The proposed viscometer has oscillations about a fixed point. Based on the perfect similitude between translational motion and rotation motion around a fixed axis, the method of finding the drag force is applicable to the new viscometer. The progress brought by the new proposed method is that the equation of the model is described by a series of parameters that allow the amplification/ attenuation of the effect of specific forces occurring in the dynamic system, and thus the model is more flexible.

The infrastructure used for this work was partially supported by the project "Integrated Center for research, development and innovation in Advanced Materials, Nanotechnologies, and Distributed Systems for fabrication and control", Contract No. 671/09.04.2015, Sectoral Operational Program for Increase of the Economic Competitiveness co-funded from the European Regional Development Fund.

\section{References}

1. G. G. Stokes, Trans. Cambridge Phil. Soc., IX, 8 (1850)

2. H. Lamb, Hydrodynamics 6th ed. (Cambridge University Press1994)

3. L. D. Landau, E. M. Lifshitz, Fluid mechanics, 2-nd Ed. (Pergamon Press 1987)

4. A. Cameron, The principles of lubrication, (John Wiley \& Sons Inc 3rd edition 1981)

5. S. V. Gupta, Viscometry for Liquids (Springer Int. Publ. Switzerland 2014)

6. M. K. Nagarajan, Canadian J of Chemistry, 46, 1969 (1968)

7. R. A. Nelson, M. G. Olsson, Am J Phys 54 (2), 112 (1986)

8. F. C. Ciornei, S. Alaci, Fasc. Manag. Tech. Eng. XIII-1, 67 (2014) 